\title{
All-human microphysical model of metastasis therapy
}

\author{
Sarah E Wheeler', Jeffrey T Borenstein², Amanda M Clark', Mohammad R Ebrahimkhani³, Ira J Fox, Linda Griffith³, \\ Walker Inman³, Douglas Lauffenburger ${ }^{3}$, Transon Nguyen², Venkateswaran C Pillai ${ }^{5}$, Rachelle Prantil-Baun², Donna B Stolz ${ }^{6}$, \\ Donald Taylor ${ }^{1,7}$, Theresa Ulrich ${ }^{3}$, Raman Venkataramanan ${ }^{5}$, Alan Wells $s^{* 1,4,7,8}$ and Carissa Young ${ }^{3}$
}

\begin{abstract}
The vast majority of cancer mortalities result from distant metastases. The metastatic microenvironment provides unique protection to ectopic tumors as the primary tumors often respond to specific agents. Although significant interventional progress has been made on primary tumors, the lack of relevant accessible model in vitro systems in which to study metastases has plagued metastatic therapeutic development - particularly among micrometastases. A real-time, all-human model of metastatic seeding and cancer cells that recapitulate metastatic growth and can be probed in real time by a variety of measures and challenges would provide a critical window into the pathophysiology of metastasis and pharmacology of metastatic tumor resistance. To achieve this we are advancing our microscale bioreactor that incorporates human hepatocytes, human nonparenchymal liver cells, and human breast cancer cells to mimic the hepatic niche in three dimensions with functional tissue. This bioreactor is instrumented with oxygen sensors, micropumps capable of generating diurnally varying profiles of nutrients and hormones, while enabling real-time sampling. Since the liver is a major metastatic site for a wide variety of carcinomas and other tumors, this bioreactor uniquely allows us to more accurately recreate the human metastatic microenvironment and probe the paracrine effects between the liver parenchyma and metastatic cells. Further, as the liver is the principal site of xenobiotic metabolism, this reactor will help us investigate the chemotherapeutic response within a metabolically challenged liver microenvironment. This model is anticipated to yield markers of metastatic behavior and pharmacologic metabolism that will enable better clinical monitoring, and will guide the design of clinical studies to understand drug efficacy and safety in cancer therapeutics. This highly instrumented bioreactor format, hosting a growing tumor within a microenvironment and monitoring its responses, is readily transferable to other organs, giving this work impact beyond the liver.
\end{abstract}

Keywords: liver, mammary carcinoma, microenvironment, tumor dissemination

\section{Introduction}

Most cancer-related mortality is due to distant metastases. Metastasis is a multistep process whereby cells from the primary tumor invade or migrate through surrounding tissues and barrier matrices, disseminate via the vasculature (hematologic or lymphatic), arrest and extravasate at the metastatic niche, and ultimately survive and outgrow in this ectopic environment. Ectopic-site survival and subsequent proliferative outgrowth is the rate-limiting step in clinically evident disease [1,2]. Distant metastases are generally more resistant to

*Correspondence: wellsa@upmc.edu

'Department of Pathology, University of Pittsburgh, 3550 Terrace Street,

S713 Scaife Hall, Pittsburgh, PA 15261, USA

Full list of author information is available at the end of the article treatments than the primary tumor [3], underscoring the need to develop more rational therapeutic approaches based on the molecular pathophysiology in the metastatic microenvironment [4].

Clinically undetectable metastases have serious implications for cancer patients; approximately one-third of women suffered a metastatic relapse within 5 years post lumpectomy $[5,6]$. This late emergence implies that tumor cells disseminate early and survive undetected in ectopic sites [5,6]. Numerous three-dimensional models of tumor cells capture a subset of tumor behaviors [7-11]. A significant gap exists in investigating how metastatic nodules interact with the host tissue due to the inherent small-scale dimensions of most microfluidic devices, poor disease recapitulation by cell lines, and lack of a primary cell environment. There is an urgent need for integrated in vitro systems that can support the initial 
micrometastatic nodules [12] to develop rational approaches to target growing cancer cells and promote clinically undetectable micrometastases towards a dormant state [13]. This process occurs over days to weeks and current endpoint analyses provide correlative, not mechanistic, insights and are mainly limited to carcinoma cell behavior. The microphysiological system proposed is evaluable over weeks, and is comprised of all human modifiable components with programmable inputs of modifiers that allow definition of the required signals for the microenvironment.

The liver as a target tissue is ideal because it is a preferred site of metastasis for many tumors (breast, lung, colon, prostate, brain, melanomas). The liver is also the major organ for drug metabolism (both activation and detoxification), a significant factor in determining efficacy and limiting toxicities in cancer therapeutics. Hepatic tissue can thus be used to develop and determine the efficacy of anticancer agents and regimens [12,14]. The liver is also one of the primary sites of systemic regulation of nutrients and hormones associated with circadian rhythms and immune system function. These nutrients, hormones, and modulators impact the metabolic functioning of the liver and the responsiveness of tumor cells $[15,16]$. Chronic disruption of circadian rhythms is correlated with changes in immune regulators $[17,18]$, and reductions in nutrients may provide better tolerance of high-dose chemotherapy [19]. Although toxicity of some chemotherapeutic agents is linked to circadian timing of delivery in patients [20], it is unknown whether chronobiological dosing is more effective in treating micrometastases due to inability to detect these small tumors in the clinical setting. A large gap exists in linking the complex metastatic microenvironment to molecular signals that change on a diurnal basis at both systemic and local levels [21-23]. This model will yield markers of metastatic behavior that enable better clinical monitoring, and will guide the design of clinical studies to understand efficacy.

\section{Technology for this work \\ Platform}

The perfused microreactor system (LiverChip; Zyoxel Ltd, Oxford, UK) we are using allows for tissues with high-level structural organization, functional complexity, ease of use, adequate samples for assays and weeks of culture while avoiding materials that adsorb steroid hormones and drugs [24]. This project dovetails with a parallel effort on a DARPA (Defense Advanced Research Projects Agency (USA))-funded Microphysiological Systems program (PI: L Griffith, MIT), where a fluidic circuit is being applied to a broader platform of 10 different integrated microphysiological systems on a platform, enabling the study of gut/ liver effects on metastases.
To achieve temporal control of medium components, we invoke inlet micropumps to dose insulin/glucagon, glucose/fructose, cortisol, and chemotherapeutics into the reactor in tandem with a fluid control system that infuses fresh basal medium and removes waste. Bioreactors are also instrumented with oxygen measurement systems based on ruthenium microprobes. This highly instrumented three-dimensional culture format hosting a growing tumor and monitoring its responses is readily transferable to other organs.

\section{Cells}

Fresh human hepatocytes and a full complement of nonparenchymal cells offer the ability to evaluate the role of the diverse populations in the metastatic tumor microenvironment in an all-human system. Cryopreserved hepatocytes and Kuppfer cells are also evaluated. To study metastatic nodules in the liver we use representative breast cancer cell lines (MDA MB 231, MCF-7, and BT474), control epithelial cells (human mammary epithelial cells), as well as primary breast carcinoma explants. These cell sources allow us to closely model the metastatic niche of the liver in an unprecedented manner.

\section{Modeling}

Prediction of disease phenotypes and therapeutic effects based on genomic variation has proved elusive. To understand how the deregulation of cellular networks underlies complex disease states, such as cancer, an alternative systems-based approach will elucidate cellular responses to external stimuli. Predictive mathematical models are constructed by introducing the system (that is, cells) to perturbations or cues, which result in multiple cell states. Phenotypic responses are measured, enabling quantitative, multivariate computational models to evaluate how cells will respond to new combinations of cues $[25,26]$. From a systems biology perspective, signaling networks are identified that in turn facilitate the evaluation of therapeutic targets and modulate systemic effects [27,28]. Methods of multivariate analyses allow the integration of complex data (for example, cytokines, chemokines, and growth factors, acute phase proteins, and metabolites) resulting from the growth of small metastatic nodules, both to glean possible signatures of early metastatic disease and response to therapies as well as to identify therapeutic targets.

\section{Key questions addressed}

Do diurnal changes within host liver tissue alter the phenotypic behavior of the tumor cells compared with standard culture?

Noninvasive measurements (capabilities noted in Figure 1) that reveal information about the evolution of cell signaling networks, coupled with appropriate computational 


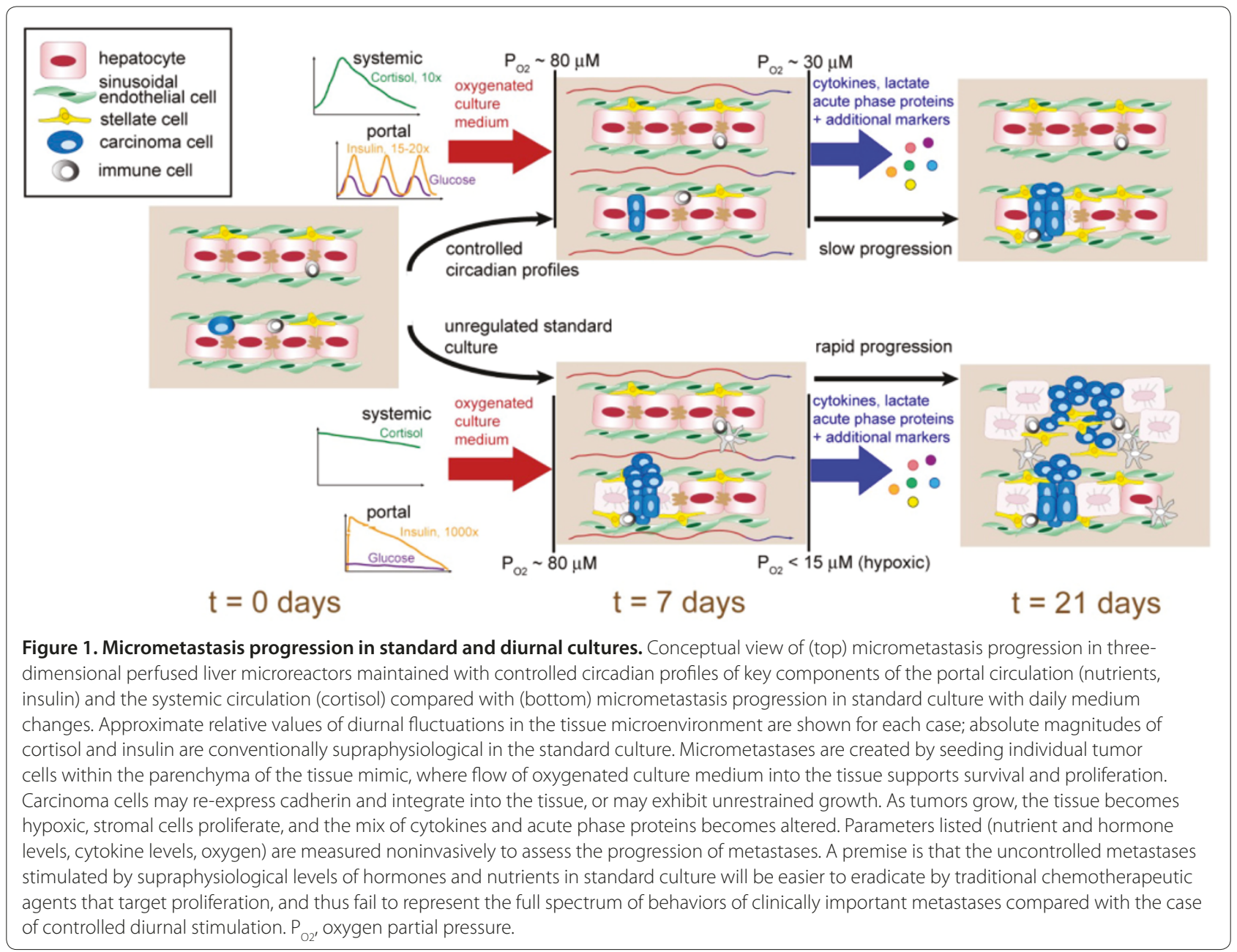

analysis, will provide a foundation for comparison with clinical observations. We previously demonstrated Ecadherin coupling between carcinoma cells and hepatocytes, and continued uncontrolled growth of other cancer cells in the population $[12,14]$. The latter phenomenon may arise from stimulation of insulin-like growth factor-1 receptor by the supraphysiological concentrations of insulin in standard hepatocellular cultures or the inflammatory cytokines due to matrix stiffening, or even the matrix stiffness itself. Diurnal control of nutrients and hormones will affect both the cancer cell and host tissue phenotypes to give rise to both macroscopic and molecular behaviors that are different from those observed in standard culture (Figure 1). An attenuated growth pattern in diurnal control cultures compared with standard culture may reflect a more physiological condition (metastatic dormancy) that will be related to experimentally testable mechanisms (for example, differences in the activation of insulin-like growth factor-1 receptor). Simultaneously sampling oxygen, metabolic, and cytokine measurements will yield insights that include crosstalk between tumor and host tissue, early markers of drug efficacy and toxicity, and therapeutic targets.

\section{Do chemotherapeutic toxicological effects on liver parallel clinically reported toxicological effects? Is efficacy of chemotherapy agents against metastatic tumors influenced by diurnal control of metabolism and hormones?}

Hepatotoxicity of first-line, nontargeted chemotherapy agents (doxorubicin, carboplatin, and so forth) and newer targeted drugs (lapatinib) is well known and managed clinically due to their efficacy in primary cancer treatment. The liver bioreactor intimately links tumor efficacy with hepatic metabolism of the agent allowing for the identification of drug combinations that may exhibit synergistic or antagonistic effects that are difficult to identify in preclinical trials. Metastases likely alter metabolism of chemotherapeutics by hepatocyte crowding and loss as well as by the signals produced by the cancer cells themselves [29]. These reciprocal paracrine 
effects likely alter both efficacy and toxicity/detoxification and are further influenced by diurnal changes in the bioactive hormones and nutrients that feed into the liver.

\section{Do mild inflammatory states of liver stimulate tumor growth and alter efficacy of chemotherapeutics?}

The mechanisms for metastatic dormancy and emergence remain unknown but a proposed explanation is that inflammatory cytokines and matrix components drive the cancer cells into a proliferative state [5]. Inflammation in the liver can come from in situ insult or portal circulation that has bacterial inflammatory initiators from the gut microbiome (among others). In the assessment of how inflammatory cues affect the tumor response we will use lipopolysaccharide as a cue [30] at low chronic doses, challenge the tissue with chemotherapy, and integrate these data into our systems biology models. We can then explore gut-liver interactions directly on our microphysiologic systems platform. The chemoresponsiveness is unpredicatable because the greater proliferative fraction should increase chemotherapy sensitivity while the inflammatory milieu should promote resistance, making this model vital for better understanding of metastatic disease therapies.

\section{Future needs and research directions}

From this work we anticipate effective solutions to augment the existing cell culture models throughout the drug development pipeline - target identification, validation, preclinical efficacy, and safety - and shift substantial risk from human clinical trials back to the preclinical development stage. We focus on occult metastases as these represent substantial challenges in preclinical development due to the complexities of identifying such nodules and monitoring their response to therapies in the clinic. We anticipate linking this model to other physiological systems (intestine, cardiovascular, pancreatic) that both regulate and are regulated by the liver to frontload the risk from human and animal subjects to in vitro systems. Additionally we envision that this platform can have substantial impact on the study of the mechanisms influencing breast cancer metastatic dormancy, emergence, proliferation and chemoresponsiveness under heretofore elusive conditions. This robust in vitro model in conjunction with systems biology will better predict disease response to new combinations of cues and treatments allowing for the identification and testing of effective therapeutic targets.

\section{Competing interests}

AW holds a patent on LiverChip now being commercialized by Zyoxel Ltd (Oxford, UK). LG holds a patent on LiverChip now being commercialized by Zyoxel Ltd, and received consulting fees paid by Zyoxel Ltd in 2012 but has no current relationship. Wl is a consultant to Zyoxel Ltd and holds stock in the oxygen sensor source (LucidScience; Boston, MA, USA). The remaining authors declare that they have no competing interests.

\section{Acknowledgements}

Funding for publication of this article came from grants $\mathrm{NIH}$ 1UH2TR000496-01 (All-human Microphysical Model of Metastasis Therapy) and DARPA-BAA-11-73 (Microphysiological Systems: W911NF-12-2-0039 Barrier-Immune-Organ: Microphysiology, Microenvironment Engineered Tissue Construct Systems (BIO-MIMETICS))

\section{Declarations}

Publication of this supplement has not been supported by sponsorship. Articles have undergone the journal's standard review process. The Editors declare that they have no competing interests.

This article has been published as part of Stem Cell Research \& Therapy Volume 4 Supplement 1, 2013: Stem cells on bioengineered microphysiological platforms for disease modeling and drug testing. The full contents of the supplement are available online at http://www.stemcellres.com/supplements/4/S1.

\section{Author details}

'Department of Pathology, University of Pittsburgh, S418 BST, 200 Lothrop Street, Pittsburgh, PA 15261, USA. ${ }^{2}$ Charles Stark Draper Laboratory, 555 Technology Square, Cambridge, MA 02139, USA. ${ }^{3}$ Department of Biological Engineering, Massachusetts Institute of Technology, 77 Massachusetts Ave, Rm 16-267, Cambridge, MA 02139, USA. ${ }^{4}$ McGowan Institute for Regenerative Medicine, University of Pittsburgh, 3025 East Carson Street Room 226, Pittsburgh, PA 15203, USA. ${ }^{5}$ Department of Pharmaceutical Sciences, University of Pittsburgh, 1104 Salk Hall, 3501 Terrace Street, Pittsburgh, PA 15261, USA. ${ }^{6}$ Department of Cell Biology, Department of Surgery, University of Pittsburgh, 3500 Terrace Street, S362 Biomedical Science Tower (South), Pittsburgh, PA 15261, USA. 'Department of Bioengineering, University of Pittsburgh, 300 Technology Drive, Room 306B, Pittsburgh, PA 15219, USA. ${ }^{8}$ Pittsburg VA Medical Center, VA Pittsburgh Healthcare System, University Drive, Pittsburgh, PA 15240, USA.

Published: 20 December 2013

\section{References}

1. Cameron MD, Schmidt EE, Kerkvliet N, Nadkarni KV, Morris VL, Groom AC, Chambers AF, MacDonald IC: Temporal progression of metastasis in lung: cell survival, dormancy, and location dependence of metastatic inefficiency. Cancer Res 2000, 60:2541-2546.

2. Luzzi KJ, MacDonald IC, Schmidt EE, Kerkvliet N, Morris VL, Chambers AF, Groom AC: Multistep nature of metastatic inefficiency: dormancy of solitary cells after successful extravasation and limited survival of early micrometastases. Am J Pathol 1998, 153:865-873.

3. Gonzalez-Angulo AM, Morales-Vasquez F, Hortobagyi GN: Overview of resistance to systemic therapy in patients with breast cancer. Adv Exp Med Biol 2007, 608:1-22

4. Wells A, Yates C, Shepard CR: E-cadherin as an indicator of mesenchymal to epithelial reverting transitions during the metastatic seeding of disseminated carcinomas. Clin Exp Metastasis 2008, 25:621-628.

5. Aguirre-Ghiso JA: Models, mechanisms and clinical evidence for cancer dormancy. Nat Rev Cancer 2007, 7:834-846.

6. Naumov GN, MacDonald IC, Weinmeister PM, Kerkvliet N, Nadkarni KV, Wilson SM, Morris VL, Groom AC, Chambers AF: Persistence of solitary mammary carcinoma cells in a secondary site: a possible contributor to dormancy. Cancer Res 2002, 62:2162-2168.

7. Nelson CM, Vanduijn MM, Inman JL, Fletcher DA, Bissell MJ: Tissue geometry determines sites of mammary branching morphogenesis in organotypic cultures. Science 2006, 314:298-300.

8. Shaw KR, Wrobel CN, Brugge JS: Use of three-dimensional basement membrane cultures to model oncogene-induced changes in mammary epithelial morphogenesis. J Mammary Gland Biol Neoplasia 2004, 9:297-310.

9. Hsiao AY, Tung YC, Qu X, Patel LR, Pienta KJ, Takayama S: 384 hanging drop arrays give excellent $Z$-factors and allow versatile formation of co-culture spheroids. Biotechnol Bioeng 2012, 109:1293-1304.

10. Ridky TW, Chow JM, Wong DJ, Khavari PA: Invasive three-dimensional organotypic neoplasia from multiple normal human epithelia. Nat Med 2010, 16:1450-1455.

11. Inamdar NK, Borenstein JT: Microfluidic cell culture models for tissue engineering. Curr Opin Biotechnol 2011, 22:681-689. 
12. Yates C, Shepard CR, Papworth G, Dash A, Beer Stolz D, Tannenbaum S, Griffith L, Wells A: Novel three-dimensional organotypic liver bioreactor to directly visualize early events in metastatic progression. Adv Cancer Res 2007, 97:225-246.

13. Taylor DP, Wells JZ, Savol A, Chennubhotla C, Wells A: Modeling boundary conditions for balanced proliferation in metastatic latency. Clin Cancer Res 2013, 19:1063-1070.

14. Chao Y, Wu Q, Shepard C, Wells A: Hepatocyte induced re-expression of E-cadherin in breast and prostate cancer cells increases chemoresistance. Clin Exp Metastasis 2012, 29:39-50.

15. Gibbs JE, Blaikley J, Beesley S, Matthews L, Simpson KD, Boyce SH, Farrow SN, Else KJ, Singh D, Ray D, Loudon AS: The nuclear receptor REV-ERBalpha mediates circadian regulation of innate immunity through selective regulation of inflammatory cytokines. Proc Natl Acad Sci U S A 2012, 109:582-587.

16. Bechtold DA, Gibbs JE, Loudon AS: Circadian dysfunction in disease. Trends Pharmacol Sci 2010, 31:191-198.

17. Castanon-Cervantes O, Wu M, Ehlen JC, Paul K, Gamble KL, Johnson RL, Besing RC, Menaker M, Gewirtz AT, Davidson AJ: Dysregulation of inflammatory responses by chronic circadian disruption. J Immunol 2010, 185:5796-5805.

18. Hansen J, Stevens RG: Case-control study of shift-work and breast cancer risk in Danish nurses: impact of shift systems. Eur J Cancer 2012, 48:1722-1729.

19. Lee C, Raffaghello L, Brandhorst S, Safdie FM, Bianchi G, Martin-Montalvo A, Pistoia V, Wei M, Hwang S, Merlino A, Emionite L, de Cabo R, Longo VD: Fasting cycles retard growth of tumors and sensitize a range of cancer cell types to chemotherapy. Sci Trans/ Med 2012, 4:124ra127.

20. Ohdo S: Circadian rhythms in the CNS and peripheral clock disorders: chronopharmacological findings on antitumor drugs. J Pharmacol Sci 2007, 103:155-158.

21. Ma D, Panda S, Lin JD: Temporal orchestration of circadian autophagy rhythm by C/EBPbeta. Embo J 2011, 30:4642-4651.
22. Atwood A, DeConde R, Wang SS, Mockler TC, Sabir JS, Ideker T, Kay SA: Cell-autonomous circadian clock of hepatocytes drives rhythms in transcription and polyamine synthesis. Proc Natl Acad Sci U S A 2011, 108:18560-18565.

23. Lamia KA, Sachdeva UM, DiTacchio L, Williams EC, Alvarez JG, Egan DF, Vasquez DS, Juguilon H, Panda S, Shaw RJ, Thompson CB, Evans RM: AMPK regulates the circadian clock by cryptochrome phosphorylation and degradation. Science 2009, 326:437-440

24. Domenech M, Yu H, Warrick J, Badders NM, Meyvantsson I, Alexander CM, Beebe DJ: Cellular observations enabled by microculture: paracrine signaling and population demographics. Integr Biol (Camb) 2009, 1:267-274.

25. Janes KA, Albeck JG, Gaudet S, Sorger PK, Lauffenburger DA, Yaffe MB: A systems model of signaling identifies a molecular basis set for cytokineinduced apoptosis. Science 2005, 310:1646-1653.

26. Aldridge BB, Saez-Rodriguez J, Muhlich JL, Sorger PK, Lauffenburger DA: Fuzzy logic analysis of kinase pathway crosstalk in TNF/EGF/insulininduced signaling. PLOS Comput Biol 2009, 5:e1000340.

27. Kreeger PK, Lauffenburger DA: Cancer systems biology: a network modeling perspective. Carcinogenesis 2010, 31:2-8.

28. Ideker T, Winslow LR, Lauffenburger AD: Bioengineering and systems biology. Ann Biomed Eng 2006, 34:257-264.

29. Hirschhaeuser F, Sattler UG, Mueller-Klieser W: Lactate: a metabolic key player in cancer. Cancer Res 2011, 71:6921-6925.

30. Dash A, Inman W, Hoffmaster K, Sevidal S, Kelly J, Obach RS, Griffith LG, Tannenbaum SR: Liver tissue engineering in the evaluation of drug safety. Expert Opin Drug Metab Toxicol 2009, 5:1159-1174.

doi:10.1186/scrt372

Cite this article as: Wheeler SE, et al:: All-human microphysical model of metastasis therapy. Stem Cell Research \& Therapy 2013, 4(Suppl 1):S11. 